\title{
latrogenic Foreign Materials Associated with Retrieved Clot Tissue via Mechanical Thrombectomy
}

\author{
(D) M. Abbasi, (DD. Dai, (D) Y. Liu, (D) S. Fitzgerald, (D) R. Kadirvel, (D) L.E. Savastano, (D) H. Cloft, (DD.F. Kallmes, and (D)W. Brinjikji
}

\begin{abstract}
BACKGROUND AND PURPOSE: Hydrophilic polymers and polytetrafluoroethylene liners, commonly used in the construction of endovascular devices, occasionally separate from devices with subsequent embolization. We determined the frequency of such materials in thrombus specimens retrieved by mechanical thrombectomy in patients with stroke.
\end{abstract}

MATERIALS AND METHODS: We retrospectively reviewed H\&E-stained thrombus sections for presence and types of foreign materials. We identified 4 types of foreign materials-Type I: material was light green with refraction and had a homogeneous texture; type II: material was light gray and/or dark gray, thin, and loose or attenuated in texture; type III: material was light green with refraction, solitary in texture, irregular in shape, and was often associated with round or oval bubblelike particles and/or diffuse black particles; and type IV: material had homogeneous texture and was light pink or red. In addition, polymer materials from different layers of used mechanical thrombectomy catheters were compared with the foreign materials found in thrombus specimens.

RESULTS: A total of 101 thrombi were evaluated. Foreign materials were found in 53 (52.5\%) thrombus samples. The most common type was type I (92\%), followed by type II (30\%). The histopathologic features of the polymer materials from mechanical thrombectomy catheters were similar to the foreign materials found in thrombus specimens. The inner polytetrafluoroethylene liner and coating layer of catheters resembled type I and type II of the foreign materials, respectively.

CONCLUSIONS: Foreign polymer materials are present in approximately half of retrieved thrombi, most commonly polytetrafluoroethylene from catheter liners and less from hydrophilic coatings.

ABBREVIATIONS: ASP = aspiration; MT = mechanical thrombectomy; PTFE = polytetrafluoroethylene

$\mathbf{N}$ euroendovascular catheters are constructed with various polymers aimed at improving lubricity, including polytetrafluoroethylene (PTFE) liners and hydrophilic coatings. The benefits of these materials include low coefficients of friction, improved trackability during the endovascular procedure, easy lesion passage, and vasospasm prevention. ${ }^{1}$ However, introduction of such foreign material to the body is associated with some risks. Many reports have suggested that foreign materials could dissociate from catheters with subsequent distal embolization and microinfarctions. ${ }^{2,3}$

Received November 18, 2020; accepted after revision January 30, 2021. From the Departments of Radiology (M.A., D.D., Y.L., S.F., R.K., L.E.S., H.C., D.F.K., W.B.) and Neurosurgery (L.E.S.), Mayo Clinic, Rochester, Minnesota.

This study is supported by the National Institutes of Health grant (No. RO1 NS105853).

Please address correspondence to Daying Dai, MD, PhD, Department of Radiology, Mayo Clinic, 200 First St SW, Rochester, MN 55905; e-mail: dai.daying@mayo.edu

- Indicates open access to non-subscribers at www.ajnr.org

http://dx.doi.org/10.3174/ajnr.A7106
In the current study, we performed histologic examination of 101 thrombus specimens to evaluate how often polymer materials are liberated from catheters. All of the thrombus samples were retrieved by mechanical thrombectomy (MT) from patients with large-vessel occlusion stroke. To confirm the source of the foreign materials observed in thrombi, we examined the histologic appearance of each polymer layer of the aspiration catheters commonly used in thrombectomy, and compared the histology with that of the foreign materials observed in thrombus specimens.

\section{MATERIALS AND METHODS Patient Population}

After independent review board approval, 101 thrombus samples retrieved from patients with acute ischemic stroke from our center were collected for conventional, histopathologic evaluation. Demographic data were collected. Procedural data including thrombectomy approach (aspiration [ASP], stent retriever, and a 

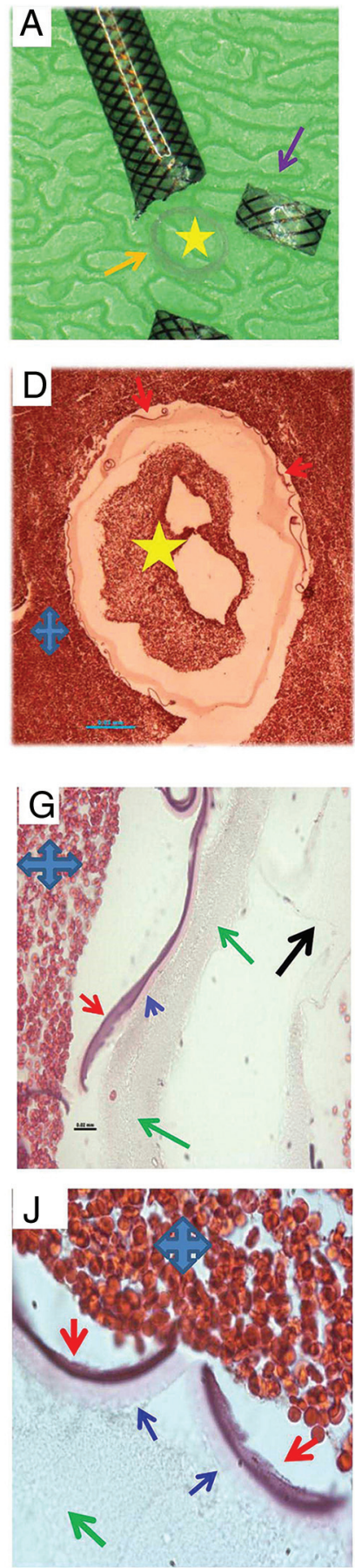
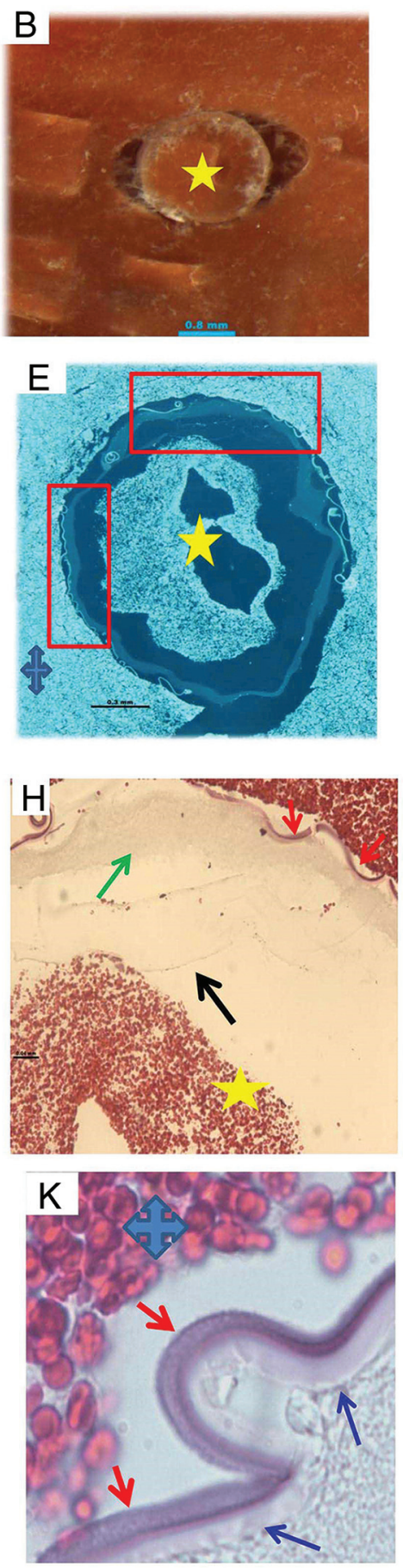
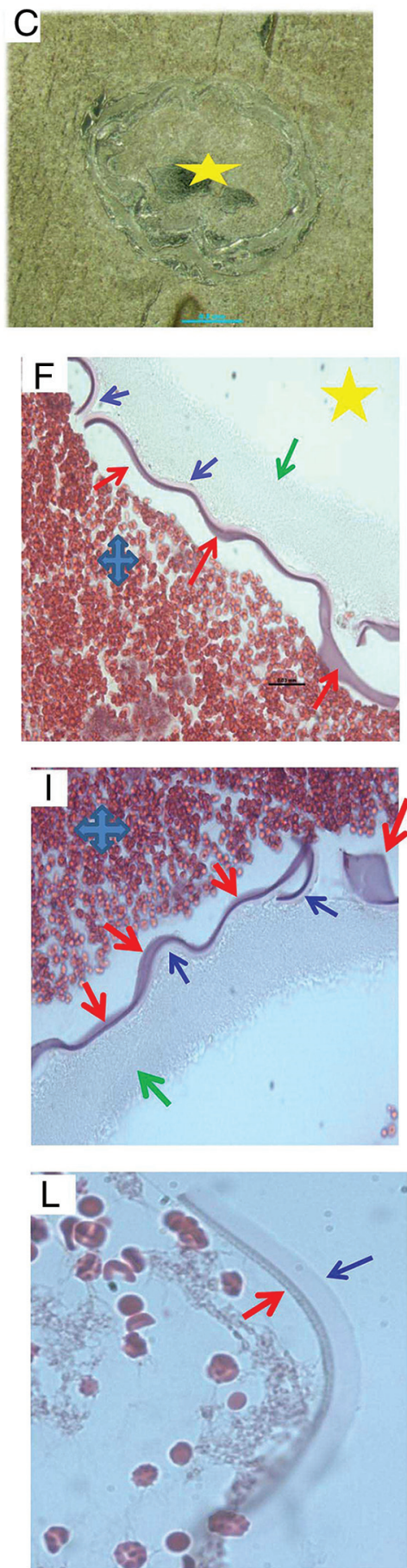

FIG 1. Histologic processing of cross-section of MT catheter. A, Macrophotograph of the Sofia catheter, showing the catheter is cut into pieces in coronary plane (arrows), with removal of metal scaffold for further histology processing (orange arrow) (asterisk: lumen side). B, Macrophotograph of catheter piece in image A mixed with clot analog tissue (asterisk: lumen side). C, Macrophotograph showing the catheter piece mixed with clot tissue embedded in paraffin after tissue processing; catheter piece remains in the coronal orientation (asterisk: lumen side). $D$, Low-magnification microphotograph of H\&E-stained catheter piece from image $C$ (blue cross: the surrounding clot; yellow asterisk: the lumen/ inner side of catheter; red arrows: outer layer surface-coating material that is next to the surrounding clot tissue). $E$, Reverse image of image $D$, showing each layer of the catheter, in relation to the lumen and outside surrounding clot tissue (yellow star: lumen side; blue cross: surrounding clot tissue). F-I, High magnification of microphotograph taken from the red rectangular area in image $E$, showing each layer of the catheter materials from the outer layer surface (red arrows), middle layer (blue arrows and green arrows) to the inner liner layer (black arrow) (H\&E, original magnification $\times 400$; yellow star: the lumen side of catheter; blue cross: the surrounding clot analog tissue). J-L, Microphotographs taken with oil lens, showing the surface-coating material (red arrows), its subsequent layer (blue arrows), and deeper layer (green arrow). The surface-coating material appears to be light gray, gray-pink, or lightly stained; attenuated or loose in texture; and with varied shape, same as the type II foreign material found in the patient clot tissue (Fig 6). The layer that is immediately underneath the coating layer appears to be homogeneous in texture, light pink, or pale in color (blue arrows). It is similar to the type IV foreign material observed in the patient clot tissue (Fig 8) (H\&E, oil lens $\times 100)$. 

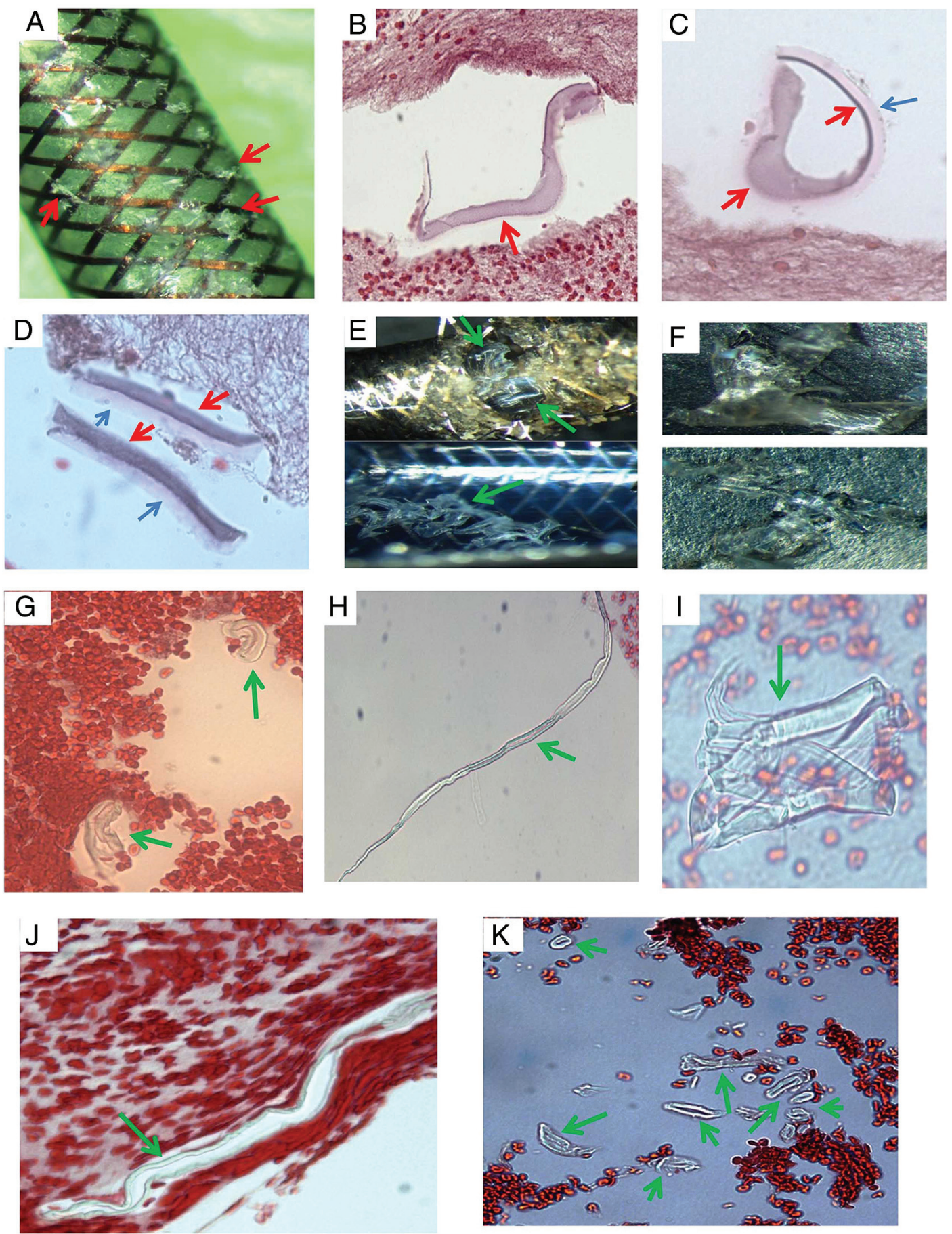

FIG 2. Histologic appearance of scratched surface and inner layer of MT catheter. A, Macrophotograph of the Sofia catheter, showing the scratched surface pieces (red arrows). B-D, Microphotograph of scratched pieces from image $A$ associated with clot analog tissue, showing the surface-coating material, which appears to be varied in shape and texture following tissue processing (red arrows), and which is similar to the material seen in the cross-section experiment, Fig 1). The layer immediately underneath the surface-coating material is also present (blue arrow), which appears to be a different shape, light pink/red, and has a homogeneous texture. It is similar to the material seen in the cross-section (Fig 1, blue arrow), and type IV foreign material (Fig 8) found in the patient clot tissue as well. E, Macrophotograph of Sofia and Fubuki catheters, showing the scratched liner layer material in situ (green arrows; upper-Sofia; lower-Fubuki). F, Macrophotograph of the manually torn off liner material pieces from image $E$, showing the similar gross appearance to that of the PTFE material (Fig 4). These pieces were crushed into small pieces for further routine, histologic processing, similar to the surface scratched material mentioned in Fig 1. G-l, Microphotograph of torn off liner pieces from $F$ (upper), showing the material that appears to be varied in shape, color, and texture because of processing. These could be tubelike, long stripe, or an irregular solid mass, with refraction and light green outline. Those features are the same as what is seen from the PTFE material (Fig 4), and the type I material found in the patient clot (Fig 5). $J$ and $K$, Representative microphotograph of scratched, torn off liner pieces from $F$ (lower), showing the material that appears to be long, or short, or small tubular in shape, and light green, as shown with the PTFE material (Fig 4) and type I foreign material found in the patient clot tissue (Fig 5). 

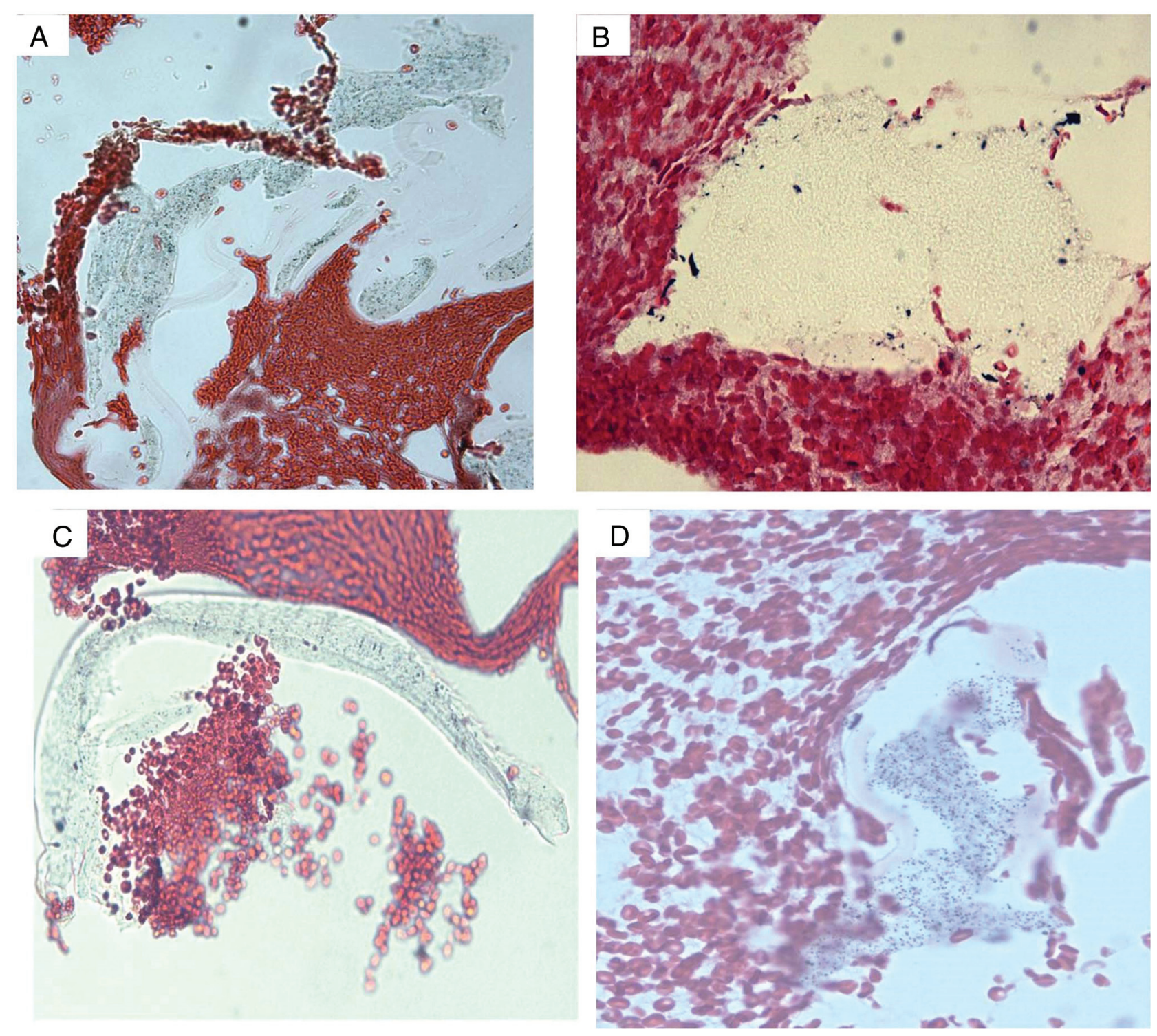

FIG 3. Representative microphotographs of scratched pieces of different layers rather than the surface or inner layer. Microphotographs showing the different materials of MT catheter, rather than the surface-coating material and inner liner materials shown in Figs 1 and 2. The similar foreign material is found in the patients' retrieved clot tissue (type III, Fig 7) ( $\mathrm{H} \& \mathrm{E}$, original magnification $\times 400)$.

combination), number of device passes, and list of devices (particularly catheters used for thrombectomy) were collected.

\section{Histopathologic Evaluation of Retrieved Thrombi}

Immediately after MT, thrombus samples were placed in $10 \%$ natural buffered formalin for at least 24 hours for fixation. After macrophotography, thrombus samples went through routine lab tissue processing as described by Fitzgerald et al. ${ }^{4}$ Samples were then embedded in paraffin and sectioned in 3- to 5- $\mu \mathrm{m}$ thickness. At least 2 serial sections from each sample were stained with H\&E. In addition, 2 serial sections were stained with Martius Scarlet Blue for clot composition analysis. ${ }^{5}$ All H\&E-stained slides were evaluated by an experienced pathologist (D.D., with more than 18 years of experience) for the presence and the types of foreign materials in thrombus samples. Based on the color and texture of foreign materials in $\mathrm{H} \& \mathrm{E}$-stained slides, 4 types of foreign materials were categorized and defined as the following types. Type I: materials were light green with refraction and had homogeneous texture. Type II: materials were light gray or and dark gray, thin, and loose or attenuated in texture. Type III: materials were light green with refraction, solitary in texture, irregular in shape, and were often associated with round or oval bubblelike particles and/or diffused black particles. Type IV: Materials had homogeneous texture, and they were light pink or red. Foreign materials observed under light microscopy were photographed and recorded for each sample. The location where the foreign material was observed was also recorded as within the clot tissue or alongside the tissue edge.

Each individual foreign material piece or pool of foreign material pieces on each slide of the patient was measured using Image-Pro 10 (Media Cybernetic). The average size (length $\times$ width) was used as representative for each type, respectively. 

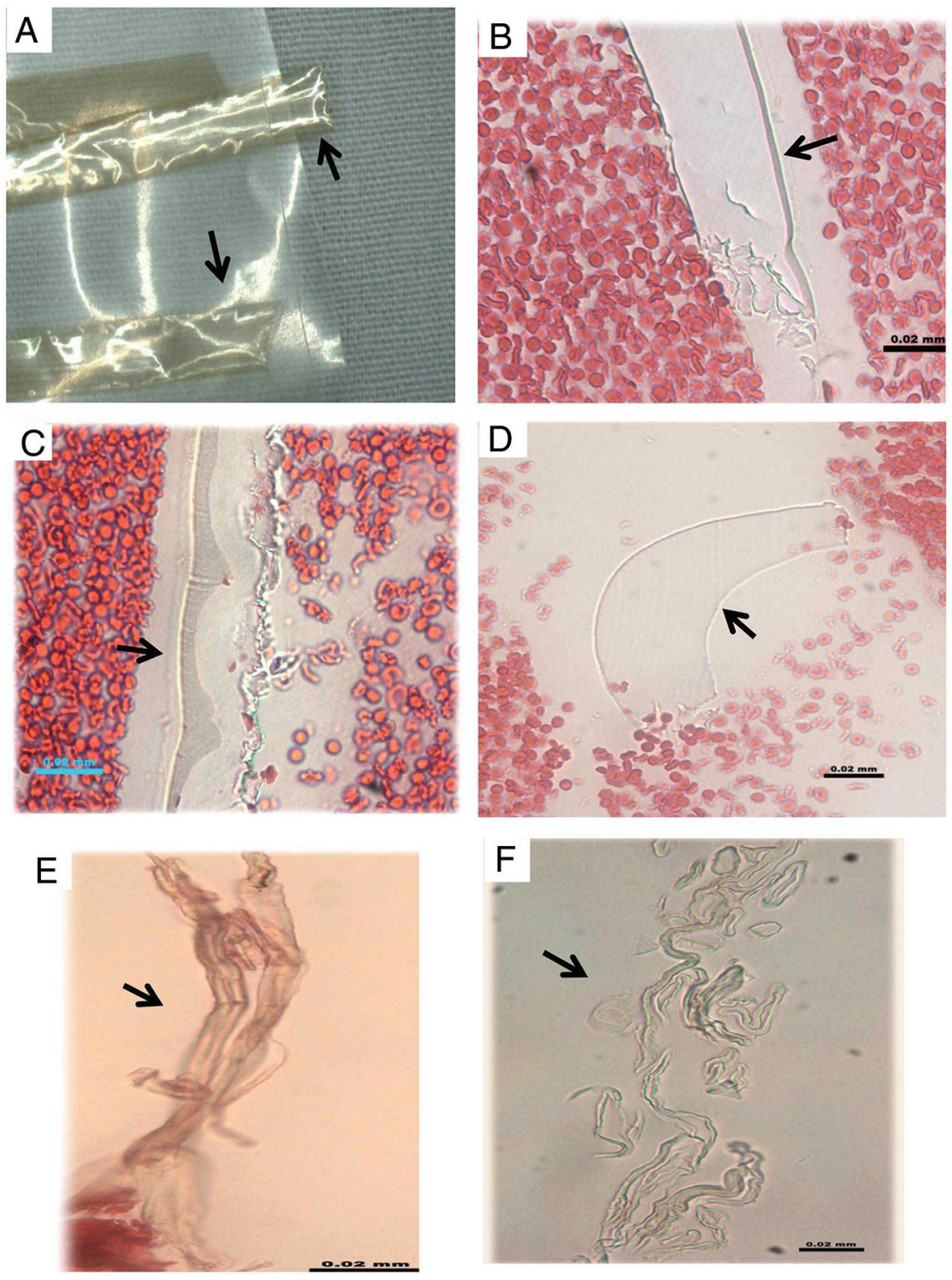

FIG 4. Histologic features of donated PTFE material. A, Macrophotograph of the PTFE liner material provided by manufacturer sponsor. $B-F$, Microphotographs of the scratched PTFE pieces in H\&E-stained slides. The material appears to have varied shape. It is either a mass with solid, homogeneous texture associated with refraction, and light green edge $(B-D)$, or long strips or tubes with light green outline $(E$ and $F)$. Those features are the same as the scratched inner layer of catheters (Fig 2), and type I material found in the patient clot tissue as well (Fig 5) (B-F, H\&E, original magnification $\times 400$ ).

\section{Histopathologic Features of Polymer Materials Used in Catheters for MT}

To determine the source of the foreign materials seen in the patients' thrombus samples, we evaluated the histologic appearance of the polymer materials of 2 commonly used catheters for MT at our institution: the Sofia suction catheter (MicroVention) and Fubuki guide catheter (Asahi Intecc). Catheters were collected after MT procedures and were carefully cleaned, dried, and sterilized. Under a dissecting microscope, the catheter wall was cut into pieces in a coronal plane, followed by removal of metal pieces (Fig 1A). The catheter pieces were then mixed with human blood in a coronal (cross) orientation, followed by a clot analog formation protocol, using a method described by Fitzgerald et al. ${ }^{6}$ The catheter piece associated with the clot analog was processed, embedded, sectioned, and stained in the same manner as the retrieved thrombi from patients (Fig 1). Each layer of polymeric materials from the catheter on the H\&E-stained slide was visually compared with the foreign materials found in the patients' thrombus specimens. Manual scratching of the catheter, layer by layer, and starting from the very outer layer to the lumen inner layer longitudinally, was also performed under dissection microscope. The scratched material pieces from each layer were mixed with clot analog, and processed in the same way as above. The materials scratched from each layer of catheter on the H\&Estained slide were also visually compared with the layer at the cross/coronal section of the catheter, and the patient clot tissue as well (Figs 2 and 3).

The PTFE material that constructs the liner layer of the catheters was also provided specifically for the current study by the manufacturer sponsor. This material was manually scratched, 

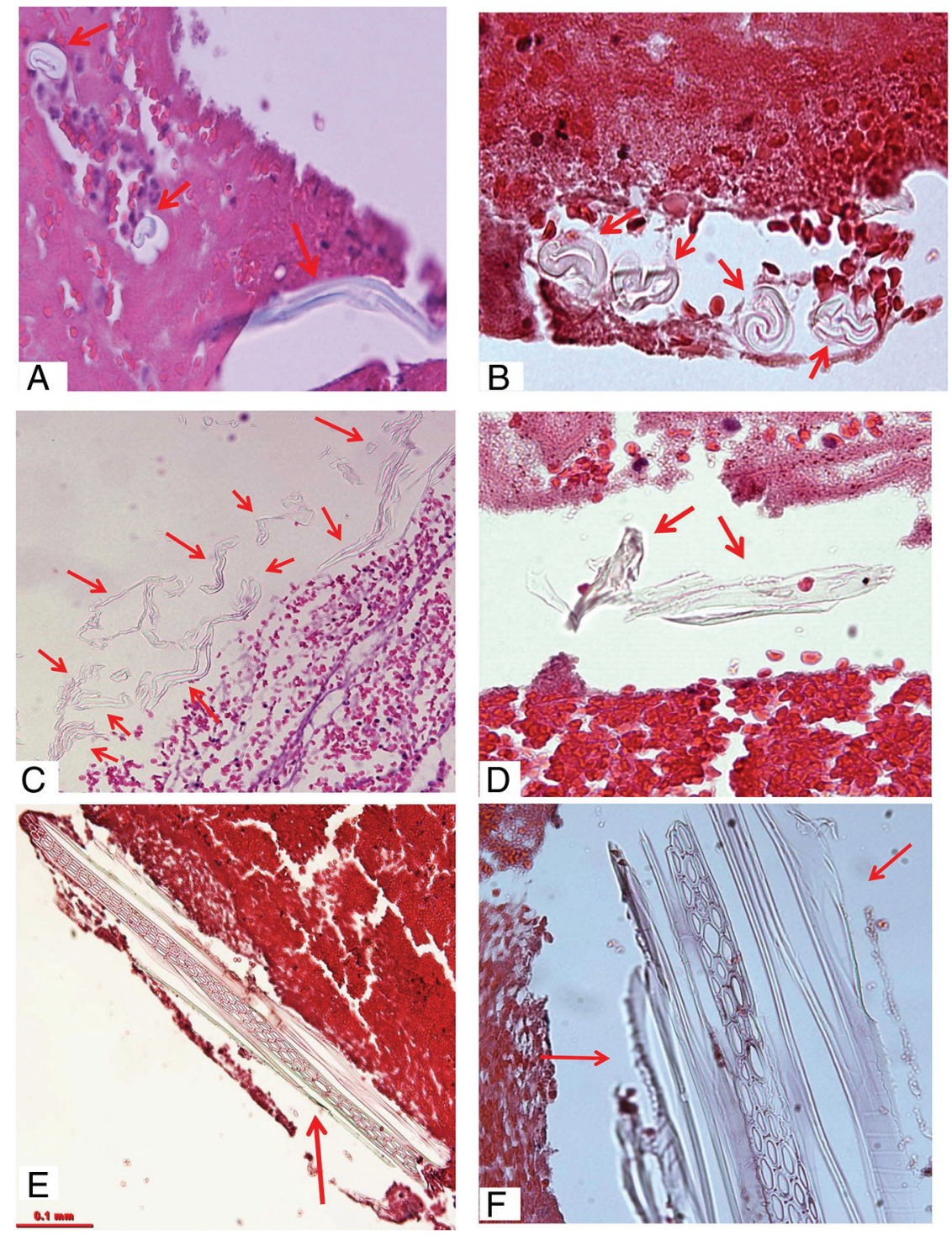

FIG 5. Representative microphotographs of type I foreign material found in patient clot tissue. A, Individual foreign material pieces separately embedded within the clot tissue (arrows). They appear to be small, ringlike, or long tubelike in shape, and light green (H\&E, original magnification $\times 400)$. $B$, The cluster of foreign material pieces (arrows). Individual pieces appear to be wormlike with refraction and a light green outline $(\mathrm{H} \& \mathrm{E}$, original magnification $\times 400)$. C, Multiple pieces of material associated with clot tissue. They are long or short stripes or pieces, with refraction, and green outline (arrows) (H\&E, original magnification $\times 200$ ). $D$, Irregular, solid pieces of material, with refraction, and light green outline $(\mathrm{H} \& E$, original magnification $\times 400)$. $E$ and $F$, Large piece of polymer material surrounded with clot tissue (arrows). It appears to be long strips or ringlike circle, and solid homogeneous mass with refraction, with light green outline (H\&E, original magnification $\times 200[E], \times 400[F])$.

torn off into pieces, and subsequently processed for histologic evaluation like the catheters mentioned above. The morphologic features (Fig 4) of the PTFE liner were also compared with foreign material found in the patient clot tissue and the material scratched from the catheters.

\section{Association of Thrombus Composition with the Presence of Foreign Materials}

Our experienced pathologist (D.D., with 18 years of lab-working experience) also evaluated the correlation between thrombus composition and the presence of foreign materials to see whether a higher percentage of any of the components, particularly fibrin and platelet, results in a higher likelihood of materials release from catheters. In addition, we evaluated any inflammatory response to the foreign materials presented in the clot. Acute inflammatory response was defined as the presence of polymorphonuclear neutrophil, monocytes reacting to foreign materials. ${ }^{7}$

\section{Association of the Presence of Foreign Material with MT Approach and Angiographic \\ Outcome}

The association of an MT approach (ASP or a combination of ASP and stent retriever) with the angiographic outcome using the final modified TICI reperfusion score and the number of device passes with the presence of foreign material found in the clot tissue was also evaluated.

\section{Statistical Analysis}

All statistical analyses were performed with the SPSS software package, v.24 for Windows (IBM). For descriptive analysis, categoric variables were presented as a percentage. The student $t$ test and $\chi^{2}$ test were used for inferential analysis. The level of significance was $P<.05$.

\section{RESULTS}

A total of 101 thrombi were retrieved from patients. The mean age for patients was 65.9 years (SD, 11.9) years. Fifty-six patients (55\%) were men. The most common thrombectomy approach was ASP in $58 \%$ of patients, followed by combination of ASP and stent retrievers (31\%). The average number of passes to achieve successful revascularization (TICI $2 \mathrm{~b}$ ) was 2.2 (SD, 1.2). Overall, the median number of passes was $2(\mathrm{IQR}=1-2)$.

\section{Histopathologic Features of Foreign Materials Found in Thrombi}

Foreign materials were found in 53 (52.5\%) of 101 thrombus samples. The foreign materials were categorized into 4 types 

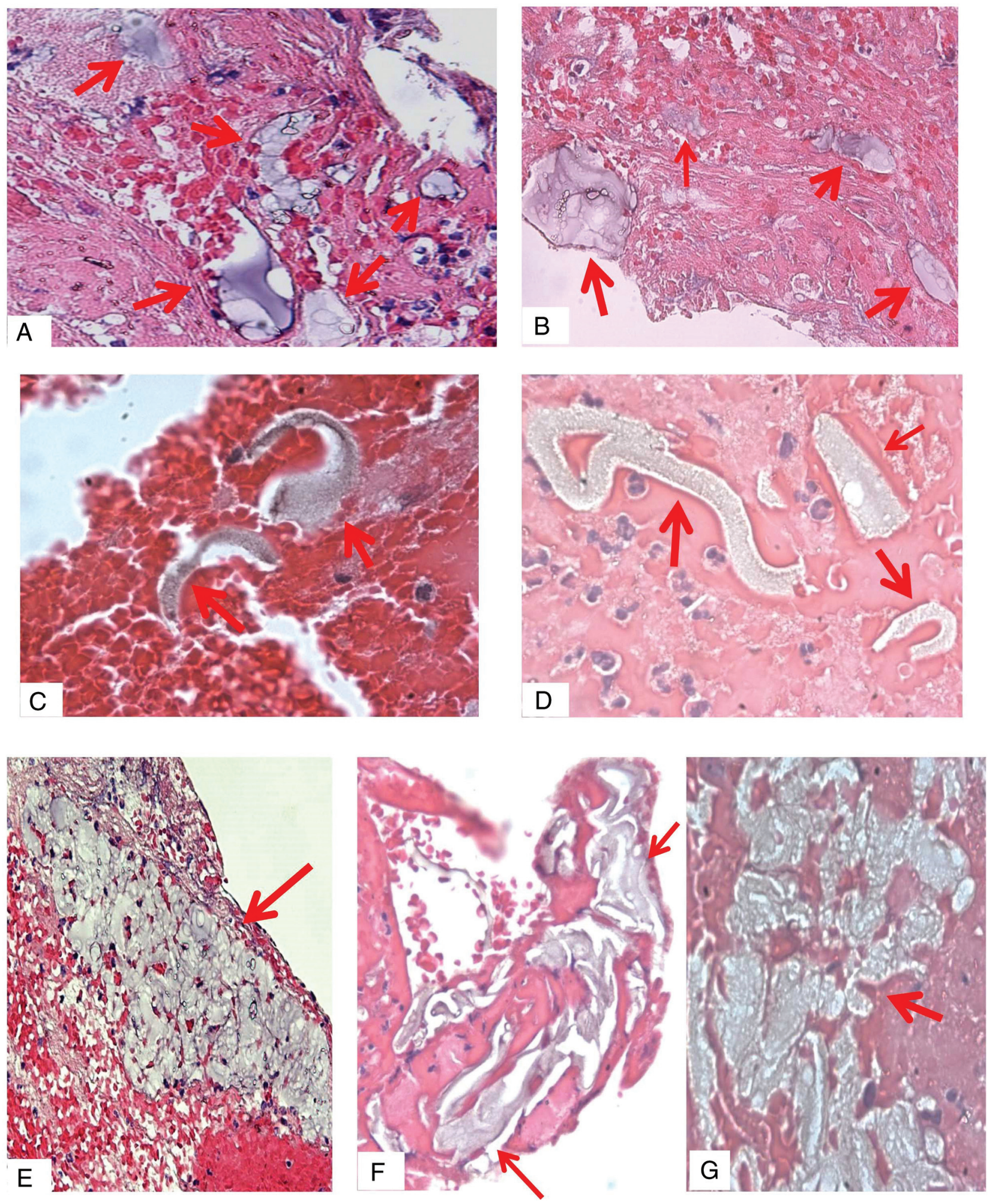

FIG 6. Representative microphotographs of type II foreign material found in patient clot tissue. $A$ and $B$, Multiple pieces of foreign material embedded within clot tissue (arrows). They appear to be light gray or dark gray; thin or attenuated in texture (H\&E, original magnification $\times 400)$. $C$ and $D$, Foreign material pieces embedded within clot tissue, which appear to be denser and solid in texture (arrows), compared with that in $A$ and $B$. They are wormlike or irregular in shape, gray or light gray (H\&E, original magnification oil lens $\times 100)$. E-G, Cluster of the foreign material pieces, as a pool surrounded with clot tissue (H\&E, original magnification $\times 200[E], \times 400[F$ and $G]$ ). 

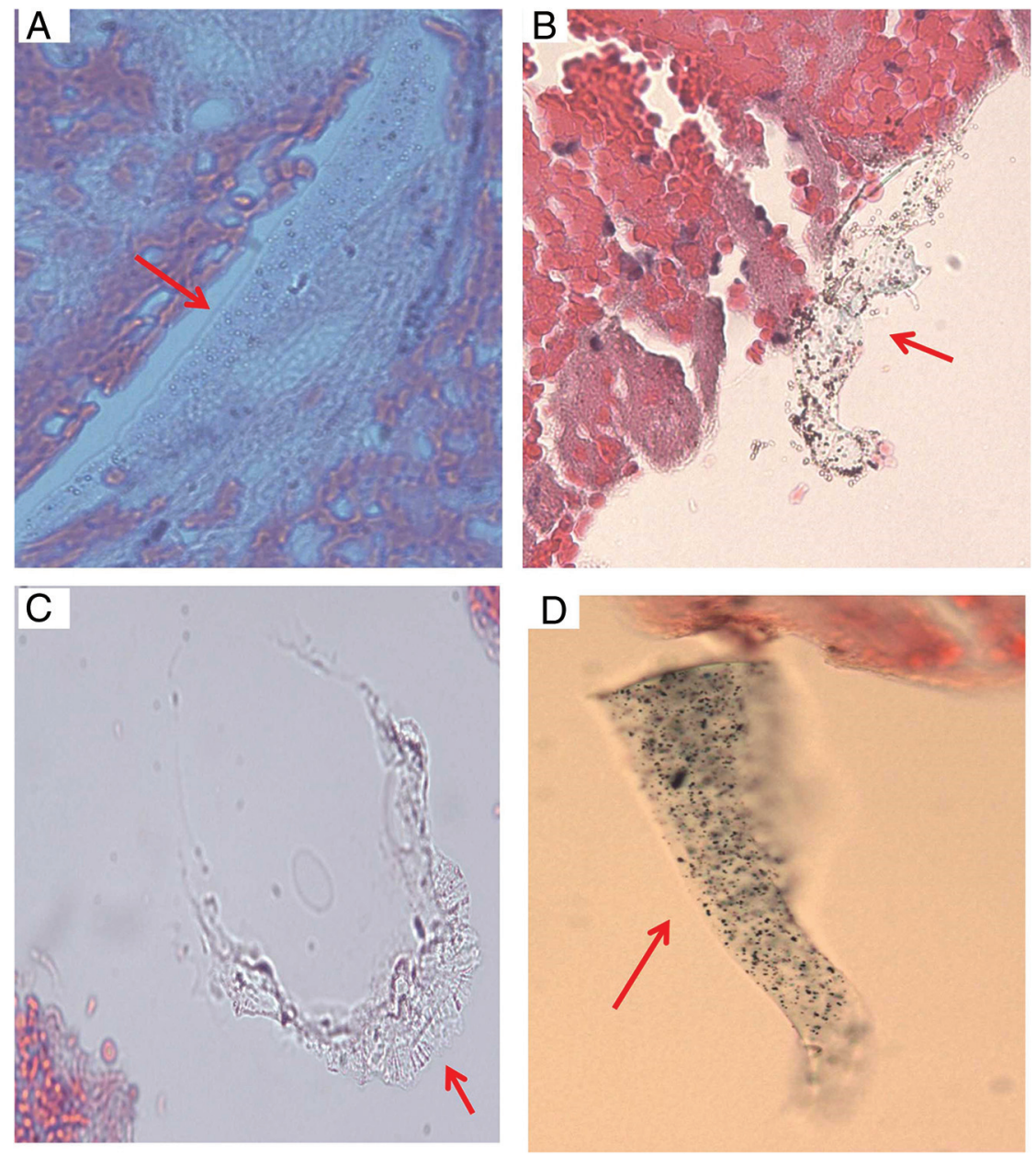

FIG 7. Representative microphotographs of type III foreign material found in patient clot tissue. A, A single, long, oval-like piece of foreign material embedded within clot tissue (arrow). The material is associated with multiple bubblelike particles, which are refracted under light microscopy. B, A single piece of irregular foreign material attaching to the clot tissue (arrow). It appears to be light green, associated dark/black and bubblelike, with refraction particles. C, Irregular piece of foreign material at the gap of clot tissue (arrow). It appears to be solid, homogeneous in texture with refraction, and light green. $D$, A piece of green, solid foreign material, associated diffused black, dotlike particles (arrow) (A$D, H \& E$, original magnification $\times 400)$. These materials presented in $A-D$ are similar to the materials scratched from the catheters (Fig 3).

based on their microscopic appearance. The prevalence of each type of foreign materials was as follows:

Type I material was found in 49 (92\%) of 53 cases that had foreign materials (Fig 5). The materials presented in different shapes, including tubular, short/long stripe, wormlike, or irregular pieces. They were scattered, single pieces, or clusters of varied size pieces. They were either located alongside the tissue border, at the gap between tissue masses, or were embedded within the clot tissue. These pieces measured from 6 to $1340 \mu \mathrm{m}$ in length. The average size of this type of material was $64.9 \times 16.3 \mu \mathrm{m}$ (length $\times$ width).

Type II materials were found in 16 (30\%) of 53 patient samples (Fig 6). They were embedded within thrombus tissue and presented as scattered or isolated separated pieces. They also gathered together as clusters. The pieces had various shapes, including cloudlike, honeycomblike, wormlike, cylinderlike, or irregular. They measured 5 to $690 \mu \mathrm{m}$ in length. The average dimensions of type II material were $110.5 \times 44.5 \mu \mathrm{m}$ (length $\times$ width).

Type III materials were found in $11(21 \%)$ of 53 samples and were solitary in texture and irregular in shape (Fig 7). The material pieces were either located alongside the tissue section or embedded within the tissue. They measured 8 to $270 \mu \mathrm{m}$ in length. The measured average size for this material was $93.8 \times 34 \mu \mathrm{m}$ (length $\times$ width).

Type IV materials were found in 8 (15\%) of 53 samples (Fig 8). They were homogeneous in texture and lightly stained. They presented as snake or wormlike in shape. They were either located at gaps between tissues or embedded within thrombus tissue. They were measured 10 to $330 \mu \mathrm{m}$ in length. The average size of this type of material was $126.4 \times 16.5 \mu \mathrm{m}$ (length $\times$ width).

Of the 53 samples that had polymer foreign material in the clot tissue, 29 (55\%) cases showed type I material only in their clot tissue; and 20 (38\%) cases had type I associated with other different types of materials. The frequency of each type of foreign material mentioned above, and the combinations of different types of material found in the clots are presented in Fig 9.

We found no inflammatory reactions to the foreign materials in thrombus specimens indicating the interaction of foreign materials with the tissue was acute.

\section{Histopathologic Features of Polymer Material from Catheters Used for MT}

The layers of the polymer material constructing the catheters are shown in Figs 1-3. The morphologic appearances of the PTFE liner material are shown in Fig 4. All types of foreign materials found in the thrombus samples retrieved from patients (Figs 5-8) in the current study demonstrated the same or similar morphologic features of materials that constructed the catheters in the scratched experiments (Figs 1-4). The inner layer of both catheters (Fig 2) and the inner PTFE material ${ }^{4}$ both demonstrated the similar morphologic appearances of type I material found in the patient clots (Fig 5). The very outer surface coating material (Figs 

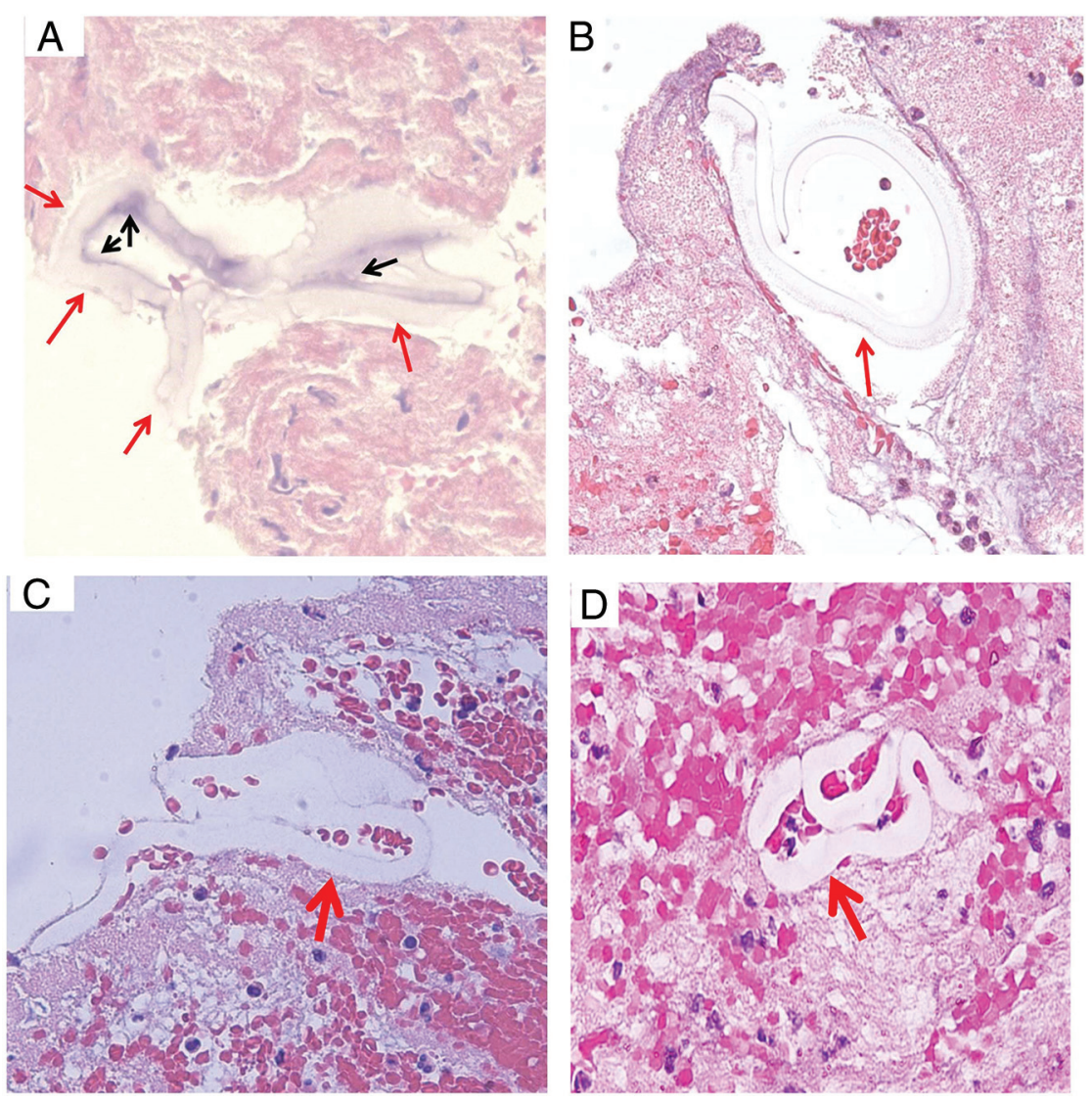

FIG 8. Representative microphotographs of type IV foreign material found in patient clot tissue. Different shapes of the type IV foreign material (red arrows) found in the clot tissue retrieved with MT. The foreign material is surrounded with clot tissue, appears to be homogeneous in texture, wormlike or snakelike in shape, and stained lightly. The residual, surface-coating material remains visible $(A$, black arrows) $(A-D, H \& E$, original magnification $\times 400)$.
In addition, the number of thrombectomy passes was not related to the presence of foreign materials $(P=.34)$.

\section{DISCUSSION}

Our study has a number of clinically relevant findings. First, we found that around half of the retrieved emboli had foreign material particles that shared similar morphologic features of polymer materials found in the neurovascular catheters, confirming the frequent release of debris from catheters during stroke intervention. Second, the polymer materials dislodging from catheters could be as long as $1.3 \mathrm{~mm}$, and most of the particles were longer than $10 \mu \mathrm{m}$, the normal size of human capillaries. This phenomenon raises the concern of microinfarctions once these particles detach from the thrombus and embolize to the blood stream. Third, our results showed that the prevalence of foreign materials in thrombus specimens is not associated with the number of thrombectomy passes or thrombus composition. Fourth, we found no inflammatory reaction to foreign materials identified in the retrieved thrombi, suggesting the material interaction with tissue was an acute event and was from the MT procedure itself. Notably, the predominant foreign material was the PTFE liner, rather than the hydrophilic outer coating. The catheters including microcatheters, aspiration catheter, sheath, or 1 and 2) showed similar morphologic features of type II polymer material found in the retrieved thrombus samples (Fig 6). The rest of the other materials (type III and IV) found in the patient clot tissue (Figs 7 and 8) also had the similar features of other materials scratched from the catheters (Figs 1-3).

\section{Association of Thrombus Composition with the Presence Foreign Materials}

The thrombus specimens had average histologic composition of $52.8 \%$ red blood cells, $3.5 \%$ white blood cells, $26.5 \%$ fibrin, and $15.0 \%$ platelets. The presence of foreign materials was not associated with thrombus composition $(P>.05)$.

\section{Association of the Presence of Foreign Material with MT Approach and Angiographic Outcome}

No association was found between the presence of foreign material and MT approaches $(P=.58)$ (Fig 10). In addition, there was no relation between final modified TICI reperfusion score and presence of foreign material in clot samples $(P=.17)$. A statistically significant correlation was not found between different types of polymer and final modified TICI reperfusion score $(P=.49)$. any other devices with known PTFE liner, which were used with the MT operation (stent retriever, ASP, or stent retriever and ASP), could be one of the contributors. The shedding or tearing of the device lining and/or coating materials could happen when the stent retriever, guiding wire guiding catheters, and/or any other catheters were advancing to the target clot tissue; or as they were pulled back into the access catheter to be retrieved or dragged out of the patient's body. The circumstances mentioned here would have friction applied to the catheter materials resulting in their dislodgement.

This present study complements the current body of medical literature which, over the past decade, provides evidence that shearing of hydrophilic polymer coating materials is an under-recognized phenomenon during neuroendovascular procedures. In addition, our study found that the PTFE liner material was more frequently seen in the clot tissue associated with neurovascular procedure than the hydrophilic coating polymer. We present our results to enhance the awareness of this phenomenon among neurointerventionists and those studying retrieved thrombi. Our results are particularly important given the growing interest in the study of retrieved emboli in the stroke literature, as these materials could be identified during standard histologic analysis. Based on 


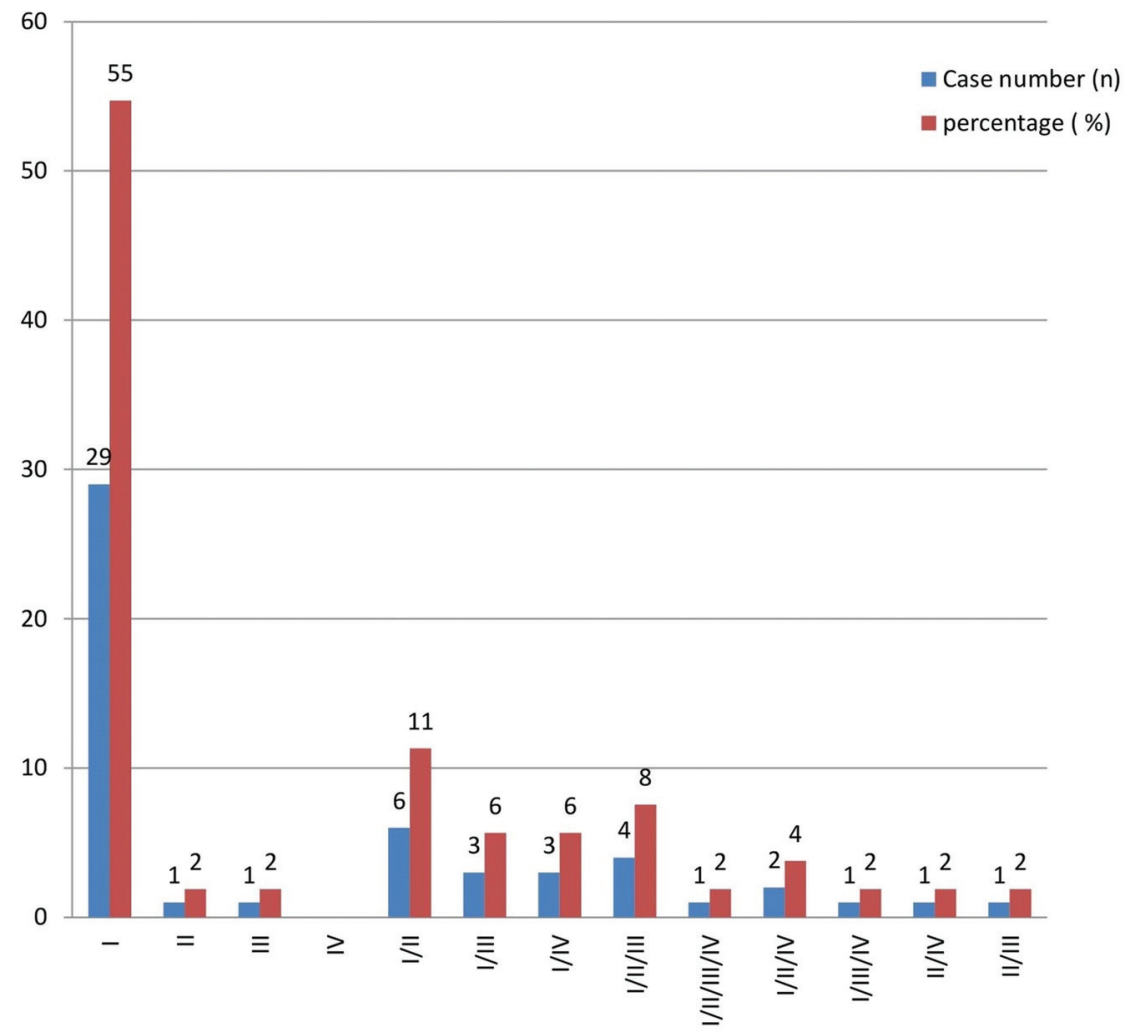

Foreign materials and their combination presented in clot tissue

FIG 9. Foreign materials and varied combinations of different types found in patients with stroke treated with MT.

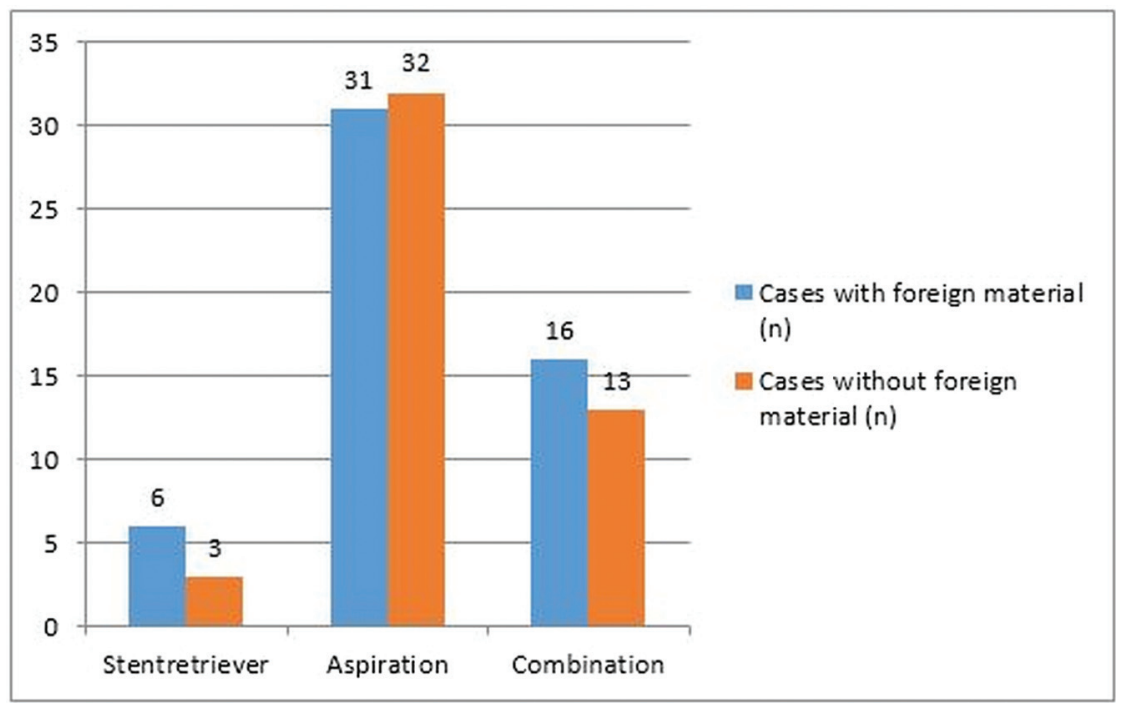

FIG 10. The association between foreign materials and MT approach. the clinical consequences associated with coating materials detaching from catheters include fever, inflammation, arteritis, vasculopathy, focal or multifocal hemorrhage, transient ischemia or microinfarction, and thrombosis, which can have a long-lasting effect and may result in irreversible tissue damage. ${ }^{2,3,8,12,13}$ However, we suspect that the embolization of microscopic polymer materials is a relatively common phenomenon that does not routinely result in major clinical complications.

Distal embolization during MT is often clinically attributed to atheroemboli or thromboemboli; however, polymer embolism is now a well-documented event associated with endovascular procedures. One could speculate that the embolization of polymer materials may lead to occlusion of small intracranial arteries and subsequent microinfarctions. Chronic inflammation associated with the presence of foreign materials could potentially complicate microinfarctions further. Therefore, polymer embolism should be considered in the differential of unexpected ischemic or inflammatory events, as well as unexplained vasculopathies happening after endovascular procedures in patients. ${ }^{14}$

Our study has limitations. We do not have routine follow-up data on patients to see whether dislodged foreign materials can cause any significant complications. We suspect that the neurologic consequences from emboli of these polymers are generally limited. We did not quantify the number of foreign material particles in thrombus specimens because of technical limitations. Although harvested polymer materials from the Sofia, Fubuki catheter, and PTFE liners show similarity to the foreign materials found in the patients' retrieved thrombus this study, we still need to establish the significance of these dissociated materials in postinterventional clinical settings.

Numerous studies have reported neurologic tissue injuries due to the presence of various catheter coatings. ${ }^{1-3,8-11}$ At present, specimens, chemistry analysis and Transmission Electron Microscopy examination are still necessary to show the details of the foreign material found in the patient clot tissue in the future. We assume the dislodging of catheter material could 
happen along with device advancing during the clot removal, or when the device/clot was pulled back through catheters both inside and outside the human body. However, the current study cannot answer exactly when the shedding happened. In addition, our study sample size was small and included only a few brands of catheters currently used in clinical settings at our institution. More studies with larger sample sizes are needed to evaluate the occurrence rate and type of polymer materials that could detach from different catheters.

\section{CONCLUSIONS}

Foreign polymer materials were found in around half of the thrombi collected from MT inpatients with large-vessel occlusion stroke. Our findings suggest that polymer materials are often separated from endovascular devices during thrombectomy; however, the clinical significance of this finding remains to be elucidated.

Disclosures: Yang Liu—UNRELATED: Consultancy: Endovascular Engineering Inc.; Comments: Scientific Board Member; Employment: Mayo Clinic Rochester; Luis Savastano-UNRELATED: Board Membership: Endovascular Engineering and VerAvanti; David Kallmes_UNRELATED: Board Membership: NoNO Inc, Vesalio Inc.; Comments: Data and Safety Monitoring Board membership; Grants/Grants Pending: Medtronic, MicroVention, Insera Therapeutics; Comments: Research support; Stock/Stock Options: Superior Medical Experts LLC, Marblehead Medical LLC; Comments: Founder/Owner; Other: Triticum Inc.; Comments: Advisory Board; Waleed Brinjikji-RELATED: Grant: National Institute of Neurological Disorders and Stroke, National Institutes of Health, *Money paid to institution.

\section{REFERENCES}

1. Fealey ME, Edwards WD, Giannini C, et al. Complications of endovascular polymers associated with vascular introducer sheaths and metallic coils in 3 patients, with literature review. Am J Surg Pathol 2008;32:1310-16 CrossRef Medline

2. Mehta RI, Mehta RI, Solis OE, et al. Hydrophilic polymer emboli: an under-recognized iatrogenic cause of ischemia and infarct. Mod Pathol 2010;23:921-30 CrossRef Medline
3. Mehta RI, Mehta RI, Fishbein MC, et al. Intravascular polymer material after coil embolization of a giant cerebral aneurysm. Hum Pathol 2009;40:1803-07 CrossRef Medline

4. Fitzgerald S, Wang S, Dai D, et al. Orbit image analysis machine learning software can be used for the histological quantification of acute ischemic stroke blood clots. PloS One 2019;14:e225841 CrossRef Medline

5. Fitzgerald ST, Wang S, Dai D, et al. Platelet-rich clots as identified by Martius Scarlet Blue staining are isodense on NCCT. J Neurointerv Surg 2019;11:1145-49 CrossRef Medline

6. Fitzgerald S, Dai D, Douglas AS, et al. Abstract WP108: novel human acute ischemic stroke blood clot analogues for in-vitro testing. Available at https://www.ahajournals.org/doi/abs/10.1161/str.51. suppl_1.WP108. Accessed October 1, 2020

7. Ward PA, Lentsch $\mathrm{AB}$. The acute inflammatory response and its regulation. Arch Surg 1999;134:666-69 CrossRef Medline

8. Mehta RI, Mehta RI, Choi JM, et al. Hydrophilic polymer embolism and associated vasculopathy of the lung: prevalence in a retrospective autopsy study. Hum Pathol 2015;46:191-201 CrossRef Medline

9. Shapiro M, Ollenschleger M, Baccin C, et al. Foreign body emboli following cerebrovascular interventions: clinical, radiographic, and histopathologic features. AJNR Am J Neuroradiol 2015;36:2121-26 CrossRef Medline

10. Barnwell SL, D'Agostino AN, Shapiro SL, et al. Foreign bodies in small arteries after use of an infusion microcatheter. AJNR Am J Neuroradiol 1997;18:1886-89 Medline

11. Mehta RI, Mehta RI. Polymer-induced central nervous system complications following vascular procedures: spectrum of iatrogenic injuries and review of outcomes. Hum Pathol 2016;53:178-90 CrossRef Medline

12. Kozak M, Adams DR, Ioffreda MD, et al. Sterile inflammation associated with transradial catheterization and hydrophilic sheaths. Catheter Cardiovasc Interv 2003;59:207-13 CrossRef Medline

13. Mehta RI, Rai AT, Vos JA, et al. Intrathrombus polymer coating deposition: a pilot study of 91 patients undergoing endovascular therapy for acute large vessel stroke, Part I: histologic frequency. $J$ Neurointerv Surg 2019;11:1191-96 CrossRef Medline

14. Mehta RI, Mehta RI. Hydrophilic polymer embolism: an update for physicians. Am J Med 2017;130:e287-e90 CrossRef Medline 